\title{
Restaurações endocrown: uma revisão de literatura
}

\author{
Endocrown restorations: \\ a literature review
}

\section{Maria Vitória Medina Bettencourt ${ }^{1}$ (1) Adna Barros Ismerim² Emilena Maria Castor Xisto Lima ${ }^{3}$ (1) Adriana Oliveira Carvalho 4 (1)}

\author{
1Escola Bahiana de Medicina e Saúde Pública (Salvador). Bahia, Brasil. vitoriamedina22@gmail.com \\ Universidade Estadual do Sudoeste da Bahia (Jequié). Bahia, Brasil. adnaodonto@yahoo.com.br \\ ${ }^{3}$ Escola Bahiana de Medicina e Saúde Pública (Salvador). Universidade Federal da Bahia (Salvador). Bahia, Brasil. emilenalima@gmail.com \\ ${ }^{4}$ Autora para correspondência. Escola Bahiana de Medicina e Saúde Pública (Salvador). Universidade Federal de Feira de Santana (Feira de Santana). \\ Bahia, Brasil. adrianacarvalho@bahiana.edu.br
}

RESUMO | INTRODUÇÃO: As restaurações endocrown têm sido uma técnica de restauração alternativa para dentes tratados endodonticamente destruídos extensivamente. São restaurações protéticas que possuem retenção macromecânica dentro da câmara pulpar de um dente despolpado e microrretenção com técnicas de cimentação adesiva na câmara e nas bordas da cavidade. É um procedimento restaurador menos traumático e proporciona melhor manutenção do remanescente dentário quando comparado à técnica com retentores intrarradiculares, pois não requerem desobstrução do conduto, com resultados estéticos e funcionais satisfatórios em estudos de longo prazo. OBJETIVO: O objetivo deste trabalho é revisar a literatura sobre as restaurações endocrown, bem como seu funcionamento clínico. METODOLOGIA: A revisão foi realizada por meio de levantamento bibliográfico online de artigos científicos nas plataformas Pubmed, Scielo e Google Scholar por meio dos descritores: dente não vital, prótese dentária, reabilitação oral e cerâmica, respeitando o prazo de 1999 a 2020, além de capítulos de livros de referência na prática odontológica. CONSIDERAÇõES FINAIS: Concluiu-se que o uso da restauração endocrown é uma alternativa confiável para restaurar molares extensamente destruídos endodonticamente tratados, agregando diversas vantagens do ponto de vista funcional e estético. Porém, mais estudos clínicos ainda são necessários para que o uso em pré-molares e dentes anteriores possa ser realizado, com segurança e sucesso, em longo prazo.

PALAVRAS-CHAVE: Dente não vital. Prótese dentária. Reabilitação bucal. Cerâmica.
ABSTRACT | INTRODUCTION: Through the advent of adhesive dentistry and invasive minimally preparations, endocrown restorations have been an alternative restorative technique for extensively destroyed endodontically treated teeth. They are prosthetic restorations with macro mechanical retention inside the pulp chamber of a pulped tooth and micro-retention with adhesive cementation techniques in the chamber and on the edges of the cavity. It is a less traumatic restorative procedure and provides better maintenance of the dental remnant when compared to the technique with intraarticular retainers because they do not require unblocking of the conduit, with satisfactory aesthetic and functional results in long-term studies. OBJECTIVE: This work aims to review the literature on endocrown restorations, as well as their clinical functioning. METHODOLOGY: The review was carried out through an online bibliographic survey of scientific articles on the platforms Pubmed, Scielo, and Google Scholar through the descriptors: non-vital tooth, dental prosthesis, oral rehabilitation, and ceramic, respecting the time limit from 1999 to 2020, furthermore to chapters of reference books in dental practice. FINAL CONSIDERATIONS: It was concluded that the use of endocrown restoration is a reliable alternative to restore extensively destroyed endodontically treated molars, adding several advantages from functional and aesthetic points of view. However, further clinical studies are still needed so that premolars and anterior teeth can be accomplished safely and successfully in the long term.

KEYWORDS: Non-vital tooth. Dental prosthesis. Oral rehabilitation. Ceramic.

Como citar este artigo: Bettencourt MVM, Carvalho AO, Ismerim AB, Lima EMCX. Restaurações endocrown: uma revisão de literatura. 2021;12(2):105-114. http://dx.doi.org/10.17267/2596-3368dentistry. v12i 2.3678 


\section{Introdução}

A reabilitação de dentes tratados endodonticamente ainda é um grande desafio para o cirurgião-dentista. Na maioria das vezes, há uma extensa perda de estrutura dentária, tornando o dente fragilizado e, em consequência, mais suscetível a fraturas. ${ }^{1}$ A técnica restauradora a ser utilizada para dentes tratados endodonticamente extensamente destruídos precisa ser planejada com cautela, a fim de garantir sucesso e longevidade da restauração.?

O uso de retentores intrarradiculares associados a coroa total ainda é bastante utilizado na reabilitação de dentes tratados endodonticamente extensamente destruídos, seja por meio de núcleos metálicos fundidos ou núcleos de preenchimento associados a pinos de fibra. Esses métodos, apesar de possuírem boa relevância clínica, necessitam de um preparo do conduto radicular, o que pode aumentar ainda mais o enfraquecimento do remanescente dentário ${ }^{3}$ e, consequentemente, o risco de fraturas e perfurações radiculares, além de uma possível contaminação do sistema endodôntico. ${ }^{4}$

Núcleos metálicos fundidos, apesar de serem adaptados ao conduto, possuem alta rigidez por conta do material utilizado, não sendo compatível com o módulo de elasticidade da dentina, o que pode levar ao aumento do risco de fratura irreversível, além da possibilidade de corrosão em razão do uso de metal. Já o pino de fibra de vidro pré-fabricado, ainda que possua elasticidade compatível com a dentina, gera uma maior espessura de película do cimento empregado, bem como a formação de bolhas, já que o pino não se adapta perfeitamente ao conduto, diminuindo a resistência à fratura. $., 5,5,6$

Alguns estudos $s^{7-9}$ têm confirmado que molares tratados endodonticamente restaurados sem pinos possuem resistência a fratura e modos de falha similares àqueles restaurados com o uso de pinos, o que sugere que os mesmos não são necessários. A partir deste panorama, e com o conhecimento de que preparos minimamente invasivos que preservam o máximo de estrutura dentária devem ser priorizados, as restaurações endocrown foram introduzidas como tratamento alternativo de reabilitação de molares tratados endodonticamente extensamente destruídos. O termo endocrown foi descrito em 1999 por Blind e Mormann ${ }^{12}$ como uma coroa protética que possui fixação no interior da câmera pulpar de um dente tratado endodonticamente. Neste tipo de restauração, não é necessária a desobstrução do canal radicular, pois a retenção macromecânica é obtida no interior da câmara e a cimentação com técnicas adesivas oferece microrretenção. ${ }^{10,11}$

O preparo é bastante simples e obtido rapidamente, já que o conduto radicular não necessita de preparos adicionais. É um procedimento menos traumático e que proporciona a preservação do remanescente dentário ${ }^{15}$, podendo ser considerado uma possibilidade de reabilitação para dentes tratados endodonticamente extensamente destruídos, uma alternativa aos tratamentos convencionais com retentores intrarradiculares. 14

Nesse contexto, o objetivo deste trabalho é realizar uma revisão de literatura sobre as restaurações endocrown em dentes posteriores e anteriores, assim como seu funcionamento clínico.

\section{Metodologia}

O presente trabalho é um estudo de revisão de literatura a respeito do uso das restaurações endocrown como forma reabilitadora de dentes tratados endodonticamente. O início da procura de artigos iniciou-se no ano de 2019 selecionando-se material literário nos idiomas português e inglês, publicados em periódicos nacionais e internacionais através de ferramentas de busca como Scielo, PubMed, Google Acadêmico, além de consulta bibliográfica.

Os artigos selecionados respeitaram a linha de tempo de 1999 a 2020. e os descritores utilizados para a seleção dos artigos foram "Dente não vital", "prótese dentária", "reabilitação bucal", "cerâmica". Os critérios de inclusão dos artigos foram inicialmente definidos por: artigos completos em português ou inglês, com resumos disponíveis nas bases de dados citadas anteriormente nos quais a metodologia permitisse obter evidências científicas consistentes e seguras, e que possuíssem como abordagem principal a reabilitação de dentes tratados endodonticamente.

Os critérios de exclusão foram estabelecidos por: artigos que estivessem em outros idiomas; incompletos, portando sem evidências científicas comprovadas ou 
que não estivessem inclusos nas bases de dados utilizadas. Foram selecionados 43 artigos além dos livros "Baratieri LN. Odontologia restauradora: fundamentos e técnicas" e "Pereira JR. Retentores Intrarradiculares", livros referenciais na prática odontológica que possuem capítulos relacionados ao tema escolhido.

\section{Revisão de literatura}

\section{Fragilidade de dentes tratados endodônticamente}

De modo geral e consensual, dentes tratados endodonticamente (DTE) são considerados mais fragilizados. Extensas lesões de cárie, traumatismos ou restaurações pré-existentes geram grandes perdas da integridade estrutural do dente, fragilizando-o e aumentando seu risco de fratura. .2 Com a perda da vitalidade pulpar, o dente tende a desidratar, perdendo cerca de $9 \%$ de água livre. ${ }^{5}$ Entretanto, não existe perda significativa à tração e compressão quando comparados a dentes vitais, visto por esse viés. Estudos demonstram que não há alteração no módulo elástico ou de dureza da dentina após o tratamento endodôntico. ${ }^{16,17}$

Existem fatores inerentes ao próprio tratamento endodôntico que podem diminuir a resistência dentária. $O$ acesso à câmara pulpar requer que seu teto seja completamente removido, assim como o acesso aos canais radiculares que requer a abertura dos mesmos, com grande remoção de tecido dental, como dentina e esmalte. A perda das cristas marginais pode reduzir a resistência em até $63 \%$, podendo gerar flexão e consequente microfraturas e/ou microinfiltrações. Essas microinfiltrações podem gerar uma recontaminação do sistema radicular, sendo necessário o retratamento endodôntico., $4,13,16,18$

\section{Técnicas convencionais para restaurar DTE}

Para promover a reabilitação desses dentes tratados endodonticamente e extensamente destruídos, ainda são bastante utilizadas restaurações com auxílio de retentores intrarradiculares. Durante muitos anos, o núcleo metálico fundido (NMF) foi considerado o meIhor método reabilitador para DTE. Após parcial remoção da guta percha, o conduto é moldado e após o processo de fundição do modelo enviado, temos como resultado o NMF. Por possuir metal, esse tipo de restauração possui algumas desvantagens, tais como a possibilidade de corrosão e elevado módulo de elasticidade em comparação à dentina, levando ao risco de fraturas. A alta rigidez dos núcleos metálicos pode gerar um efeito de cunha, acarretando traumas radiculares extensos, que pode condenar o dente à exodontia. Além disso, não é um material estético, possuindo custos elevados por conta da etapa laboratorial. $2,19,20$

Considerando as desvantagens do NMF, outra forma restauradora intrarradicular é o pino de fibra de vidro pré-fabricado, que se tornou popular pela facilidade de uso, baixo custo e redução de tempo clínico por dispensar etapas laboratoriais para confecção do retentor. Estão disponíveis em várias formas e tamanhos, além de possuírem módulo de elasticidade semelhantes à dentina e fácil adesão à estrutura dentária. Apesar de possuírem essas vantagens, os pinos pré-fabricados não são moldados no conduto, o que gera uma maior espessura do agente cimentante e até mesmo formação de bolhas, levando a uma redução da resistência à fratura. $2,5,5,6,21$

Ainda existem os pinos anatômicos diretos, que são moldados no conduto a partir do reembasamento com resina composta sobre o pino pré-fabricado. Nesse método, consegue-se reduzir a espessura do cimento, aumentando resistência ao deslocamento por conta do bom imbricamento mecânico. Entretanto, o uso de materiais diferentes e não homogêneos resulta em uma maior concentração de estresse sobre o remanescente dentário, pois cada material possui módulo de elasticidade diferente. ${ }^{2,5,21}$

Para a construção de retentores intrarradiculares, seja NMF ou pino de fibra, é necessária a desobstrução da guta-percha do conduto para a correta colocação da peça. Normalmente essa desobstrução equivale ao comprimento de dois terços do remanescente dental ou metade da altura do suporte ósseo do dente em questão. Esse procedimento conseguirá apenas a retenção da restauração, não interferindo no reforço do remanescente coronal e radicular. $\mathrm{Na}$ realidade, acarretará maior enfraquecimento, por conta da perda de estrutura dentária durante o preparo, além do risco de perfuração acidental da raiz, limitando o prognóstico. ${ }^{5,22}$ 
$\mathrm{Na}$ Odontologia, o efeito férula refere-se à estrutura de dente remanescente na região cervical. Segundo o estudo de Lima et al. 7 , o efeito férula é um elemento crítico na performance de coroas de molares tratados endodonticamente, pois a presença da férula aumenta a resistência à fratura desses dentes. Neste mesmo estudo, também pôde-se notar que o uso de pinos de fibra de vidro não influenciou a resistência a fratura de coroas de molares tratados endodonticamente, sugerindo que o uso de pinos em DTE extensamente destruídos não é necessário.?

\section{Restaurações endocrown}

É consensual na Odontologia que os preparos minimamente invasivos que preservam o máximo de estrutura dental sadia são o padrão ouro, sendo o objetivo de qualquer tratamento, ainda mais em dentes que já estão severamente danificados. Considerando este fato, Blind e Mormann ${ }^{12}$, em 1999, sugeriram a técnica restauradora endocrown. Essa restauração indireta é constituída de uma única peça, sendo fixada no interior da câmara pulpar com o auxílio de técnicas adesivas, não havendo necessidade de retenção intrarradicular. Elas preservam tecido dental e limitam o seu preparo interno a área da câmara pulpar, construindo ao mesmo tempo a coroa e o preenchimento da câmara pulpar. 10,22-24

Um dos primeiros estudos acerca da restauração endocrown foi realizado em 1999 por Blind e Mormann ${ }^{12}$, quando sugeriram o uso do termo. Neste estudo in vivo, 19 restaurações deste tipo foram feitas com o sistema CEREC em 13 pacientes, sendo 4 unidades pré-molares e 15 molares. Utilizando o critério USPHS modificado, essas restaurações foram avaliadas por permanência e qualidade clínica após 2 anos de acompanhamento clínico, possuindo resultados satisfatórios e promissores em pré-molares e molares. Os autores concluíram que, através da união adesiva, as forças oclusais são melhor transmitidas pela câmara pulpar, além de conseguir a redução de passagem de microorganismos para a região apical. Dessa maneira, é essencial que a técnica adesiva seja feita corretamente para garantir uma melhor sobrevida clínica da restauração endocrown.

De um ponto de vista clínico, as restaurações endocrown possuem a vantagem de serem mais baratas e simples que as convencionais. O tempo para conclusão também é reduzido, podendo ser feita no próprio consultório caso realizadas com o método CAD/CAM. Esse tipo de restauração consegue manter o máximo de preservação do remanescente dental por não necessitarem de preparos invasivos, proporcionando um resultado eficiente e estético, em uma alternativa clinicamente viável. $.13,23,24$

Belleflamme et al. ${ }^{4}$, em 2017, após mais de vinte anos da primeira definição, propuseram a primeira classificação de restaurações endocrown, baseada na quantidade do remanescente dentário posteriormente ao preparo. Essa classificação foi designada após análise clínica do dente e/ou modelos em gesso. A classe 1 refere-se a um preparo onde pelo menos duas paredes de cúspide têm uma altura superior a metade da altura original. Classe 2 descreve um preparo onde apenas uma parede de cúspide tem uma altura superior a metade da altura original. Classe 3 descreve um preparo no qual todas as paredes de cúspide estão reduzidas em mais da metade da altura original.

Em casos de espaços interoclusais limitados, onde há uma extensa perda de tecido coronal, caso fossem realizadas restaurações de ancoragem radicular, seria necessário o uso de subestruturas metálicas ou cerâmicas sob a coroa cerâmica, que comprometeria ainda mais o pequeno espaço. Nesses casos, a restauração endocrown está indicada por justamente ser constituída de uma única estrutura totalmente cerâmica. ${ }^{25-27} \mathrm{Em}$ coroas clinicamente curtas também é sugerido o uso da endocrown, já que este fator pode interferir diretamente na retenção e estabilidade da restauração colocada. A presença de raízes pequenas, obliteradas, dilaceradas ou frágeis também propiciam o uso da técnica, já que no caso de coroas tradicionais com retentores intrarradiculares seria necessário desobstrução, aumentado a possibilidade de dano irreversível. $14,27,28$

Clavijo et al. $\underline{29}$ descreveram, através de um relato de caso, a possibilidade de uso da endocrown. Um molar superior que possuía atresia em seu conduto palatino, o que desaconselhava a utilização de retentores intrarradiculares, foi o objeto de estudo. $O$ autor concluiu que por não necessitarem de retenção intracanal, as restaurações endocrown eliminam a carga máxima sobre os canais, com maior preservação da estrutura dentária, além de serem econômicos em custo e tempo. 
Por existir o risco de descolamento, quando a efetiva adesão não pode ser assegurada, a técnica não é indicada. O mesmo é válido quando a câmara pulpar é inferior a $3 \mathrm{~mm}$ de profundidade ou se a margem cervical é inferior a $2 \mathrm{~mm}$ de largura para a maior parte da sua circunferência, esses dois fatores estariam associados a uma menor área de adesão, portanto com risco de falha. $27,28,30$

\section{Preparo}

Os preparos para endocrown possuem os mesmos princípios de restaurações do tipo inlay e onlay. Recomenda-se que as paredes axiais sejam ligeiramente expulsivas, com média de 10-12 graus; fundo da câmara pulpar plano; ângulos internos arredondados e remoção de paredes de esmalte com menos de $2 \mathrm{~mm}$ de espessura. Além disso, áreas retentivas precisam ser removidas para o adequado assentamento da peça, podendo haver o preenchimento das regiões negativas com cimento de ionômero de vidro ou resina composta, fornecendo assim uma geometria expulsiva das paredes laterais. .3, $25,28,31,32^{2}$

O término cervical precisa ser uma superfície plana e polida, sem micro-irregularidades. O mesmo deve ser supragengival para facilitar a moldagem, ajustes, cimentação e diminuição do acúmulo de placa bacteriana, que poderia levar a agressão do periodonto. Entretanto, pode seguir a margem gengival em necessidades estéticas e clínicas. O principal requisito é que todas as superfícies do preparo sejam visíveis a partir de um ponto da vista oclusal. $25,31,33$

Pode-se remover a guta-percha dos canais radiculares em até $2 \mathrm{~mm}$ para servir de recurso retentivo e aumento de resistência ao deslocamento. Essa etapa do preparo deve ser executada com um instrumento não abrasivo, com o intuito de preservar a integridade da entrada dos canais.,., $34-36$

É possível a construção do monobloco através do sistema CAD/CAM. Esse tipo de usinagem permite que a câmara pulpar seja moldada a partir do scanner intraoral, e com o uso de softwares é realizado o design e a produção das coroas do tipo endocrown. 12,25,26 No entanto, a moldagem protética com utilização de elastômeros, a exemplo da silicona de adição, também pode ser utilizada, assim como a convencional confecção da coroa protética. . $8,37^{2}$

\section{Materiais restauradores}

Por se tratar de dentes tratados endodonticamente, existe uma perda do feedback sensorial em comparação aos dentes vitais, sendo um fator de aumento da carga e estresse mastigatório. O material restaurador nesse tipo de caso necessita, então, de boas propriedades mecânicas a fim de evitar fraturas ou descolamento, já que recebem maiores forças na interface da restauração, remanescente dentário e da adesividade. ${ }^{12,35}$

Dentre a categoria de materiais estéticos restauradores disponíveis indicados para a confecção de restaurações endocrown estão as cerâmicas ácido-sensíveis. Essas cerâmicas, a exemplo do dissilicato de lítio e das cerâmicas feldspáticas reforçadas com leucita, apresentam boas propriedades mecânicas e estéticas, além de uma boa biocompatibilidade com a estrutura dentária e possibilidade de condicionamento ácido. ${ }^{24-26,37}$ Esse condicionamento, se realizado de maneira adequada, possibilita uma maior estabilidade da restauração. ${ }^{27}$ Além disso, com o uso da cimentação adesiva, existe uma menor necessidade de preparos adicionais para aumento da macrorrentenção, sendo consistente com o objetivo da odontologia minimamente invasiva e com maior preservação do remanescente dentário. Entretanto, as restaurações em cerâmica também apresentam friabilidade e suscetibilidade a fratura, além de provocar o desgaste do dente antagonista. ${ }^{24}$

Outro material estético disponível para a confecção de restaurações endocrown é a resina composta indireta. Estas são caracterizadas por possuírem propriedades mecânicas similares a da dentina. Possuem baixo módulo de elasticidade, permitindo uma maior absorção das tensões geradas durante a mastigação e baixa abrasividade ao dente antagonista. No entanto, as restaurações em resina composta são mais susceptíveis ao desgaste, à fratura, à deterioração da margem e possuem menor estabilidade de cor quando comparado ao material cerâmico. ${ }^{23}$

Mörmann et al. relataram em seu estudo in vitro que a resistência a fraturas em coroas endocrown com espessura de $5,5 \mathrm{~mm}$ na parte oclusal era duas vezes mais elevada que o de coroas de $1,5 \mathrm{~mm}$ com preparação clássica, concluindo que a resistência aumenta proporcionalmente com o aumento da espessura da restauração. Essa espessura considerada ideal varia de $3-7 \mathrm{~mm} \cdot \underline{28,34}$ 


\section{Técnicas de cimentação}

Os cimentos à base de resina demonstram possuir propriedades superiores em relação a adesão à estrutura dental, resistência ao desgaste, relativa insolubilidade no meio bucal e maior poder de união química entre as interfaces quando comparadas a métodos antecessores de cimentação. Esses cimentos possuem três tipos: quimicamente ativados, fotopolimerizáveis e dual, que associa ambos. $\underline{29}^{2,38}$

Os cimentos resinosos quimicamente ativados conseguem polimerizar uniformemente em situações clínicas onde a luz não consiga atingir todas as superfícies do material; entretanto, uma manipulação inadequada pode acarretar formação de bolhas de ar, o que cria espaços vazios na interface adesiva. A cimentação fotoativada possui a vantagem e facilidade de uso de não necessitar mistura e nem manipulação, o que evita a formação de bolhas de ar, possuindo um tempo de trabalho adequado para o assentamento e ajuste da restauração. No entanto, é necessária uma adequada fotopolimerização para que o maior grau de conversão monomérica seja atingido, sendo requisito para uma boa estabilidade e resistência ao desgaste, assim como diminuição da degradação pelo meio bucal. O cimento dual consegue combinar as vantagens dos materiais quimicamente ativados com as vantagens dos cimentos ativados por luz. A cimentação química garantirá a polimerização de áreas mais profundas, onde a luz não consegue alcançar, enquanto a fotoativação garante a rápida estabilidade da peça restauradora, permitindo a adequada conversão junto às margens. ${ }^{29,38,39}$

As peças endocrown, por serem uma peça única, preenchendo ao mesmo tempo a coroa e a câmara pulpar, possuem a absorção e passagem de luz pela peça limitada por conta de sua grande espessura, sombra e opacidade. Os cimentos de eleição na cimentação dessas peças são o resinoso quimicamente ativado ou o dual, por garantirem que a região central e mais profunda da câmara pulpar, onde a luz não consegue alcançar, tenham o grau de conversão adequado. O cimento necessita alcançar o maior grau de polimerização possível em todas as áreas, pois isso garante uma boa fixação, sucesso e longevidade clínica da restauração. É contraindicado o uso de cimento fotoativado pois a luz do aparelho fotopolimerizador não consegue ultrapassar completamente a coroa, que é espessa, impedindo uma adequada polimerização do cimento. ${ }^{29,38,40}$
Um método que auxilia previamente na melhora da cimentação adesiva é o uso do Selamento Imediato da Dentina (IDS), onde a dentina é selada imediatamente após o preparo do dente e antes da tomada de impressão, evitando sua contaminação e reduzindo a possibilidade de colapso da camada híbrida. O IDS é útil no aumento do poder de retenção e adaptabilidade da restauração, por conta do aumento da capacidade de adesão da dentina. Além disso, o método permite o maior desenvolvimento de ligação das interfaces, conseguindo proteger a dentina de microinfiltrações e vazamentos bacterianos durante as fases prévias à cimentação da restauração. $1 \frac{18}{}$

\section{Discussão}

Estudos in vitro e clínicos são fundamentais para a comprovação de hipóteses sugeridas, principalmente quando se trata de técnicas e materiais. Estudos de endocrown em molares são vastos na literatura, e é possível assegurar-se de boas evidências científicas para comprovação do seu uso. O uso em pré-molares e dentes anteriores é menos relatado na literatura, porém percebe-se o aumento no interesse de estudo em relação a essas unidades, sendo extremamente importante para que o uso clínico possa ser efetivo e difundido.

Biacchi e Basting ${ }^{10}$, através de um estudo in vitro em 2012, compararam a resistência à fratura de coroas cerâmicas indiretas convencionais, incluindo núcleos de preenchimento e pinos de fibra de vidro com endocrowns. Vinte molares inferiores hígidos foram usados, divididos igualmente nos dois grupos. O grupo com as restaurações endocrown mostrou-se mais resistente às forças de compressão quando comparadas às coroas cerâmicas convencionais associadas a pinos de fibra, sendo, portanto, uma ótima opção reabilitadora para molares tratados endodonticamente, por não necessitarem de pinos intrarradiculares e serem mais resistentes que técnicas convencionais.

Em um estudo em 2005, Blind e Mormann30, objetivaram investigar, em diferentes tipos de preparo, a taxa de sobrevida e a qualidade clínica de duzentas coroas de dentes posteriores geradas pelo sistema CAD/CAM. Foram realizados três tipos de preparo: coroa clássica, coroa reduzida e endocrown. A sobrevida nos preparos para endocrown após 15 meses tiveram como resultado em $68,8 \%$ dos pré-molares e $87,1 \%$ dos molares. 
Sendo assim, essa restauração se mostrou satisfatória apenas para molares. Os autores justificaram a insatisfação no uso em pré-molares devido a menor área de superfície disponível para o agente adesivo, justificando a falha de adesão na dentina. Esse estudo entra em desacordo ao realizado pelos mesmos em 199912, quando concluíram que a restauração seria viável em pré-molares e em molares, sendo necessária uma boa adesão. Entretanto, é importante salientar que no estudo de 1999 foram utilizadas apenas quatro unidades de pré-molares para observância clínica após 2 anos, sendo um número reduzido em relação ao segundo estudo de 2005, portanto com risco de resultados menos precisos.

Em 2013, Biacchi et al. $\underline{26}$ realizaram uma pesquisa para avaliar a substituição da retenção intrarradicular por endocrown em coroas unitárias de dentes posteriores. Com um acompanhamento de três anos, concluíram que endocrown estariam restritas à reabilitação de dentes posteriores, em especial molares, pelo viés do melhor recebimento da ação de forças oclusais, estética e área para adesão. No caso dos pré-molares, o desempenho da restauração é inferior por conta da ação de forças da mastigação. A morfologia das coroas dessas unidades tem como característica a altura maior que a largura, resultando em uma força de alavanca, o que aumenta o risco de ruptura adesiva e deslocamento.

O estudo de Biachhi e Basting10 realizado em 2012 já indicava a boa utilização das restaurações endocrown em molares tratados endodonticamente, mesmo se tratando de estudo in vitro. O estudo realizado em $2013^{26}$ após acompanhamento de três anos em pacientes concluiu que a reabilitação com endocrown estaria indicada especialmente para os molares.

Guo et al., , em 2016, no seu estudo in vitro testou o uso em pré-molares inferiores, já que os mesmos possuem forma anatômica diferente dos superiores, tendo uma câmara pulpar menor e, por conseguinte, menor área para adesão. Demonstraram não existir diferença significativa entre os pinos e as endocrown em relação a resistência à fratura. Esse resultado contrapõe o estudo de Blindl et al. ${ }^{30}$, que desaconseIha o uso em pré-molares, já que o estudo de Guo et

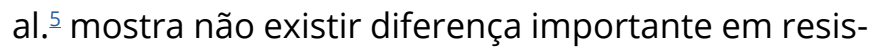
tência à fratura entre o método tradicional de reabilitação com pino de fibra de vidro e a endocrown.
Atash et al. $\underline{6}$, em 2017, após estudo em pré-molares inferiores, constatam que as restaurações endocrown se mostraram mais resistentes à fratura em relação às outras convencionais. Além disso, não mostraram maior deslocamento. Sendo assim, o curto "retentor" da endrocrown se mostra capaz de suportar maiores cargas antes de quebrar. Os resultados desse estudo demonstram a melhor praticabilidade da restauração endocrown. Entretanto, não devem ser extrapolados para um ambiente clínico real, sendo ainda necessários testes in vivo para comprovação da eficiência e evidências observadas.

Com o avanço das propriedades mecânicas das resinas compostas e resistência de união dos sistemas adesivos, Magne et al..$\underline{23}$ e Carvalho et al. $\underline{24}$ testaram se seria possível utilizar a resina composta para confeccionar um núcleo de preenchimento, dando um efeito férula em molares tratados endodonticamente extensamente destruídos, sem o uso de pinos. Outra consideração era o possível uso desse núcleo de preenchimento para remover áreas retentivas do preparo endodôntico, fornecer uma geometria positiva para facilitar a retenção do provisório e diminuição da espessura da restauração (possibilitando o uso de cimentos fotoativados). Eles concluíram que independente do uso de núcleo de preenchimento positivo ou não (restaurações endocrown) todas as coroas resistiram as forças mastigatórias. Dessa forma, sugerem que, quando houver necessidade de uma maior retenção do provisório, realize-se um núcleo de preenchimento de $2 \mathrm{~mm}$ de altura para facilitar esta retenção. No entanto, de uma perspectiva clínica, a restauração endocrown possui mais vantagens práticas como: menor custo, menor tempo de confecção e não sofrem contração de polimerização inerente das resinas compostas.

Há poucos estudos na literatura referentes ao uso da restauração endocrown em dentes anteriores, o que ainda demanda maior interesse em pesquisas para que o uso possa ser melhor entendido e ser possível de uso, com maiores esclarecimentos. Neste presente trabalho, foram utilizados apenas estudos in vitro em razão da limitada quantidade de artigos disponíveis nas plataformas de pesquisa.

No estudo in vitro realizado por Ramirez-Sebastià et al. ${ }^{41}$, em 2014, com utilização de incisivos centrais superiores, foi possível concluir que o aumento do 
tamanho do pino não aumentou a resistência da restauração. É possível considerar, então, que pinos ou endocrown podem ser usados em dentes anteriores, já que ambos estariam seguramente posicionados. Entretanto, como já relatado, é necessário que sejam feitos mais estudos clínicos e laboratoriais para a comprovação da eficácia.

Em 2020, no estudo in vitro realizado por Silva-Sousa et al. ${ }^{42}$ caninos superiores foram estudados. Em relação à fratura, o estudo concluiu que os dentes reabilitados com pinos de fibra se mostraram mais resistentes em relação às endocrowns. Apesar disso, as endocrowns ainda se mostraram uma forma viável para a reabilitação de caninos, desde que possuam o efeito férula, já que isso propicia menor tensão na coroa, no cimento e na raiz.

O estudo de Silva-Sousa et al. ${ }^{42}$ está de acordo com o estudo de Lima et al. ${ }^{7}$, no qual o mesmo afirma que a presença da férula aumenta a resistência à fratura dos dentes restaurados, sendo um fator importante para a retenção da restauração. Isso pode ser comprovado ao ser relatado que no estudo de Silva-Sousa et al. ${ }^{42}$ nenhuma amostra sem férula dos dentes caninos sobreviveu ao teste de fadiga quando restaurados com endocrown. Paralelamente a esse resultado, os que possuíam a férula se mostraram viáveis para reabilitação em dentes anteriores.

Também em 2020, no estudo de Waaz ${ }^{43}, 20$ incisivos centrais superiores foram analisados. As amostras foram submetidas a testes térmicos e de resistência à fratura em máquina de ensaio universal. O valor médio da resistência à fratura não apresentou diferença estatisticamente significativa entre os grupos testados. Apesar disso, endocrowns de profundidade curta ( $3 \mathrm{~mm}$ ) e com $2 \mathrm{~mm}$ de remanescente coronal são mais recomendados, se possível, pois sabe-se da importância de preparos minimamente invasivos com maior conservação da estrutura dentária., ${ }^{2,10}$

Os dois estudos descritos neste trabalho que verificaram as diferentes profundidades de preparo das endocrowns tiveram resultados positivos. RamirezSebastià et al. ${ }^{41}$, de 2014, e Waaz ${ }^{43}$, de 2020, trazem resultados similares em suas pesquisas, sendo possível afirmar que a profundidade do preparo intrarradicular não traz consequências em relação a resistência à fratura em dentes anteriores.
Por meio de estudos in vitro e testes clínicos de longevidade, o uso da restauração endocrown em molares tratados endodônticamente é considerada uma alternativa altamente viável, sendo dispensável o uso de retentores intrarradiculares, e sugerido que pinos e núcleos metálicos fundidos não sejam mais necessários em molares. ${ }^{7-9}$ Ainda são requeridos mais estudos e testes para uso e confirmação da viabilidade do uso em pré-molares e dentes anteriores mais seguramente. Os resultados estão sendo promissores para uma futura utilização da técnica nessas unidades, procedimento qual vêm se mostrando uma excelente estratégia restauradora.

\section{Considerações finais}

A partir dessa revisão de literatura, foi possível concluir que as restaurações do tipo endocrown são uma alternativa confiável para restaurar molares tratados endodonticamente extensamente destruídos. A técnica agrega vantagens como menor tempo clínico, maior facilidade e maior preservação da unidade dentária, com boas perspectivas do ponto de vista funcional e estético. Em relação ao uso em pré-molares e dentes anteriores, ainda é necessário que sejam feitos mais estudos clínicos e pesquisas, pois os autores ainda entram em desacordo quanto à sua viabilidade nessas unidades.

\section{Contribuições das autoras}

Bettencourt MVM participou da coleta de dados bibliográficos e escrita do artigo. Carvalho AO participou da orientação e supervisão, escrita e revisão do manuscrito. Lima EMCX e Ismerim $A B$ contribuíram com a escrita e revisão do artigo científico.

\section{Conflitos de interesses}

Nenhum conflito financeiro, legal ou político envolvendo terceiros (governo, empresas e fundações privadas, etc.) foi declarado para nenhum aspecto do trabalho submetido (incluindo, mas não se limitando a subvenções e financiamentos, participação em conselho consultivo, desenho de estudo, preparação de manuscrito, análise estatística, etc.). 


\section{Referências}

1.Zhu Z, Dong X-Y, He S, Pan X, Tang L. Effect of Post Placement on the Restoration of Endodontically Treated Teeth: A Systematic Review. Int J Prosthodont. 2016;28(5):475-83. https://doi. org/10.11607/ijp.4120

2. Clavijo VGR, Calixto LR, Monsano R, Kabbach W, Andrade MF. Reabilitação de dentes tratados endodonticamente com pinos anatômicos indiretos de fibra de vidro. Rev dental press estét [Internet]. 2008;5(2):31-49. Disponível em: https://pesquisa. bvsalud.org/portal/resource/pt/lil-529498

3. Baratieri LN, Monteiro Junior S. Coroas posteriores endocrown. In: Baratieri LN, Monteiro Junior S, Melo TS, Ferreira KB, Hilgert LA, Schlichting LH, et al.. Odontologia restauradora: fundamentos e técnicas. São Paulo: Ed. Santos; 2010. p. 738-55.

4. Belleflamme MM, Geerts SO, Louwette MM, Grenade CF, Vanheusden AJ, Mainjot AK. No post-no core approach to restore severely damaged posterior teeth: An up to 10-year retrospective study of documented endocrown cases. J Dent. 2017;63(4):1-7. https://doi.org/10.1016/j.jdent.2017.04.009

5. Guo J, Wang Z, Li X, Sun C, Gao E, Li H. A comparison of the fracture resistances of endodontically treated mandibular premolars restored with endocrowns and glass fiber post-core retained conventional crowns. J Adv Prosthodont. 2016;8(6):48993. https://doi.org/10.4047/jap.2016.8.6.489

6. Atash R, Arab M, Duterme H, Cetik S. Comparison of resistance to fracture between three types of permanent restorations subjected to shear force: An in vitro study. J Indian Prosthodont Soc. 2017;17(3):239-49. https://doi.org/10.4103/jips.jips 2417

7. Lima AF, Spazzin AO, Galafassi D, Correr-Sobrinho L, CarliniJúnior $B$. Influence of ferrule preparation with or without glass fiber post on fracture resistance of endodontically treated teeth. J Appl Oral Sci. 2010;18(4):360-3. https://doi.org/10.1590/S1678$\underline{77572010000400007}$

8. Salameh Z, Sorrentino R, Papacchini F, Ounsi HF, Tashkandi E, Goracci C, et al. Fracture Resistance and Failure Patterns of Endodontically Treated Mandibular Molars Restored Using Resin Composite With or Without Translucent Glass Fiber Posts. J Endod. 2006;32(8):752-5. https://doi.org/10.1016/j.joen.2006.02.002

9. Fokkinga WA, Le Bell AM, Kreulen CM, Lassila LVJ, Vallittu PK, Creugers NH. Ex vivo fracture resistance of direct resin composite complete crowns with and without posts on maxillary premolars. Int Endod J. 2005; 38(4):230-7. https://doi.org/10.1111/j.13652591.2005.00941.x

10. Biacchi GR, Basting RT. Comparison of Fracture Strength of Endocrowns and Glass Fiber Post-Retained Conventional Crowns. Oper Dent. 2012;37(2):130-6. https://doi.org/10.2341/11-105-L
11. Sedrez-Porto JA, Rosa WL, Silva AF, Münchow EA, PereiraCenci T. Endocrown restorations: A systematic review and meta-analysis. J Dent. 2016; 52:8-14. https://doi.org/10.1016/j. jdent.2016.07.005

12. Bindl A, Mörmann WH. Clinical evaluation of adhesively placed Cerec endo-crowns after 2 years--preliminary results. J Adhes Dent. 1999;1(3):255-65. Citado em: PMID: 11725673

13. Rocca GT, Krejci I. Crown and post-free adhesive restorations for endodontically treated posterior teeth: from direct composite to endocrowns. Eur J Esthet Dent. 2013; 8(2):156-79. Citado em: PMID: $\underline{23712338}$

14. Poluha RL, Mello Neto CL, Sábio S. Reabilitação estética em elemento posterior: endocrown. Rev Odonto de Araçatuba [Internet]. 2015;36(1):75-81. Disponível em: https:// apcdaracatuba.com.br/revista/2015/07/12.pdf

15. Dogui H, Abdelmalek F, Amor A, Douki N. Endocrown: An Alternative Approach for Restoring Endodontically Treated Molars with Large Coronal Destruction. Case Rep Dent. 2018;2018. https://doi.org/10.1155/2018/1581952

16. Schwartz RS, Robbins JW. Post placement and restoration of endodontically treated teeth: A literature review. J Endod. 2004; 30(5):289-301. https://doi.org/10.1097/00004770-200405000$\underline{00001}$

17. Pereira JR, Kaizer OB, Veiga ANA, Ghizoni JS. Restauração de dentes tratados endodonticamente. In: Pereira JR. Retentores Intrarradiculares. São Paulo: Artes Médicas; 2011. p. 17-22

18. Magne P, Kim TH, Cascione D, Donovan TE. Immediate dentin sealing improves bond strength of indirect restorations. J Prosthet Dent. 2005; 94(6):511-9. https://doi.org/10.1016/j. prosdent.2005.10.010

19. Heydecke G, Butz F, Strub JR. Fracture strength and survival rate of endodontically treated maxillary incisors with approximal cavities after restoration with different post and core systems: An in-vitro study. J Dent. 2001; 29(6):427-33. https://doi.org/10.1016/ s0300-5712(01)00038-0

20. Sirimai S, Riis DN, Morgano SM. An in vitro study of the fracture resistance and the incidence ofvertical root fracture of pulpless teeth restored with six post-and-coresystems. J Prosthet Dent. 1999; 81(3):262-9. https://doi.org/10.1016/s00223913(99)70267-2

21. Grandini S, Sapio S, Simonetti M. Use of anatomic post and core for reconstructing an endodontically treated tooth: a case report. J Adhes Dent. 2003;5(3):243-7. Citado em: PMID: 14621246

22. Magne P, Goldberg J, Edelhoff D, Güth JF. Composite resin core buildups with and without post for the restoration of endodontically treated molars without ferrule. Oper Dent. 2016;41(1):64-75. https://doi.org/10.2341/14-258-L 
23. Magne P, Carvalho AO, Bruzi G, Anderson RE, Maia HP, Giannini M. Influence of no-ferrule and no-post buildup design on the fatigue resistance of endodontically treated molars restored with resin nanoceramic CAD/CAM crowns. Oper Dent. 2014;39(6):595-602. https://doi.org/10.2341/13-004-L

24. Carvalho AO, Bruzi G, Anderson RE, Maia HP, Giannini M, Magne $P$. Influence of adhesive core buildup designs on the resistance of endodontically treated molars restored with lithium disilicate CAD/CAM crowns. Oper Dent. 2016;41(1):76-82. https:// doi.org/10.2341/14-277-L

25. Veselinovic V, Todorovic A, Lisjak D, Lazic V. Restoring endodontically treated teeth with all-ceramic endo-crowns: Case report. Stomatol Glas Srb. 2008;55(1):54-64. https://doi. org/10.2298/SGS0801054V

26. Biacchi GR, Mello B, Basting RT. The endocrown: An alternative approach for restoring extensively damaged molars. J Esthet Restor Dent. 2013;25(6):383-90. https://doi.org/10.1111/ jerd.12065

27. Dartora NR, Ferreira MBC, Spazzin AO, Sousa Neto MD, Dartora G, Gomes EA. Endocoroa em pré-molar utilizando cerâmica reforçada por dissilicato de lítio: um relato de caso. J Oral Invest. 2017;6(2):43-9. https://doi.org/10.18256/2238510X.2017.v6i2.2265

28. Fages M, Bennasar B. The endocrown: a different type of all-ceramic reconstruction for molars. J Can Dent Assoc. 2013;79:d140. Citado em: PMID: 24309044

29. Clavijo VGR, Souza NC, Kabbach W, Calixto RL, Andrade FM, Susin HA. Endocrown restorations - an approach for non-vital posterior teeth. Clín. int. j. braz. dent. 2007;(33):246-52. Disponível em: https://pesquisa.bvsalud.org/portal/resource/pt/lil-526037

30. Bindl A, Richter B, Mörmann WH. Survival of ceramic computer-aided design/manufacturing crowns bonded to preparations with reduced macroretention geometry. Int J Prosthodont. 2005;18(3):219-24. Citado em: PMID: 15945309

31. Gresnigt MMM, Özcan M, van den Houten ML, Schipper $\mathrm{L}$, Cune MS. Fracture strength, failure type and Weibull characteristics of lithium disilicate and multiphase resin composite endocrowns under axial and lateral forces. Dent Mater. 2016;32(5):607-14. https://doi.org/10.1016/j.dental.2016.01.004

32. Tzimas K, Tsiafitsa M, Gerasimou P, Tsitrou E. Endocrown restorations for extensively damaged posterior teeth: clinical performance of three cases. Restor Dent Endod. 2018;43(4):e38. https://doi.org/10.5395/rde.2018.43.e38

33. Zavanelli AC, Zavanelli RA, Mazaro JVQ, Lemos CAA, Dias ES, Silva EV, et al. Coroas Endocrown: uma revisão de literatura e relato de caso. Arch Heal Investig. 2017;6(8):382-9. https://doi. org/10.21270/archi.v6i8.2215
34. Sevimli G, Cengiz S, Oruc MS. Endocrowns: review. J Istanb Univ Fac Dent. 2015;49(2):57-63. https://doi.org/10.17096/ jiufd.71363

35. Chang CY, Kuo JS, Lin YS, Chang YH. Fracture resistance and failure modes of CEREC endo-crowns and conventional post and core-supported CEREC crowns. J Dent Sci. 2009;4(3):110-7. https:// doi.org/10.1016/S1991-7902(09)60016-7

36. Einhorn M, DuVall N, Wajdowicz M, Brewster J, Roberts H. Preparation Ferrule Design Effect on Endocrown Failure Resistance. J Prosthodont. 2019;28(1):e237-42. https://doi. org/10.1111/jopr.12671

37. Nishimori LE, Annibelli RL, Sábio S, Oliveira e Silva C, Progiante PS, Côrrea GO. Endocrown passo a passo: do laboratório à clínica. Rev Dental Press Estét [Internet]. 2012;9(4):54-61. https:// pesquisa.bvsalud.org/portal/resource/pt/biblio-857649

38. Bernardo RT, Obici AC, Sinhoreti MAC. Efeito da ativação química ou dual na microdureza knoop de cimentos resinosos. Cienc Odontol Bras [Internet]. 2008;11(4):80-5. Disponível em: https://ojs.ict.unesp.br/index.php/cob/article/view/674/566

39. Price RB, Shortall AC, Palin WM. Contemporary issues in light curing. Oper Dent. 2014;39(1):4-14. https://doi.org/10.2341/13067-LIT

40. Ayres APA, Andre CB, Pacheco RR, Carvalho AO, Bacelar-Sá RC, Rueggeberg FA, et al. Indirect restoration thickness and time after light-activation effects on degree of conversion of resin cement. Braz Dent J. 2015;26(4):363-7. https://doi.org/10.1590/0103$64402013 \times 0024$

41. Ramirez-Sebastià $A$, Bortolotto $T$, Cattani-Lorente M, Giner L, Roig M, Krejci I. Adhesive restoration of anterior endodontically treated teeth: influence of post length on fracture strength. Clin. Oral Investig. 2014;18(2):545-54. https://doi.org/10.1007/s00784013-0978-3

42. Silva-Sousa AC, Moris ICM, Barbosa AFS, Silva-Sousa YTC, Sousa-Neto MD, Pires CRF, et al. Effect of restorative treatment with endocrown and ferrule on the mechanical behavior of anterior endodontically treated teeth: An in vitro analysis. J Mech Behav Biomed Mater. 2020;112:104019. https://doi.org/10.1016/j. jmbbm.2020.104019

43. Waaz S. Impact of Preparation Depth and Length on Fracture Resistance of Anterior Teeth Restored by Endocrowns and Post Retained Crowns. Egypt Dent J. 2020;66(1):507-16. https://doi. org/10.21608/edj.2020.79126 